Draft Version November 4, 2018

Preprint typeset using $\mathrm{IAT}_{\mathrm{E}} \mathrm{X}$ style emulateapj v. 5/2/11

\title{
FORMALDEHYDE AND METHANOL DEUTERATION IN PROTOSTARS: FOSSILES FROM A PAST FAST HIGH DENSITY PRE-COLLAPSE PHASE
}

\author{
V. Taquet $^{1}$, C. Ceccarelli ${ }^{1}$, C. Kahane ${ }^{1}$ \\ (Dated: Received - ; accepted -) \\ Draft version November 4, 2018
}

\begin{abstract}
Extremely high deuteration of several molecules have been observed around low mass protostars since a decade. Among them, formaldehyde and methanol present particularly high deuteration, with observations of abundant doubly and triply deuterated forms. Both species are thought to be mainly formed on interstellar grains during the low temperature and dense pre-collapse phase by $\mathrm{H}$ and $\mathrm{D}$ atom additions on the iced CO. We present here a theoretical study of the formaldehyde and methanol deuteration obtained with our gas-grain model, GRAINOBLE. This model takes into account the multilayer nature of the mantle and explores the robustness of the results against the uncertainties of poorly constrained chemical and surface model parameters. The comparison of the model predictions with the observations leads to two major results: i) the observed high deuteration is obtained during the last phase of the pre-collapse stage, when the density reaches $\sim 5 \times 10^{6} \mathrm{~cm}^{-3}$, and this phase is fast, lasting only several thousands years. ii) $\mathrm{D}$ and $\mathrm{H}$ abstraction and substitution reactions are crucial in making up the observed deuteration ratios; This work shows the power of chemical composition as a tool to reconstruct the past history of protostars.
\end{abstract}

\section{INTRODUCTION}

Although the deuterium elemental abundance is $1.5 \times$ $10^{-5}$ relative to hydrogen (Linsky 2003), observations carried out during the last decade have revealed high abundances of singly, doubly, and even triply deuterated molecules in low mass pre-stellar cores and Class 0 protostars (Ceccarelli et al. 2007). In this work, we focus on formaldehyde and methanol, both species likely synthesized on the grain surfaces (Tielens \& Hagen 1982; Watanabe \& Kouchi 2002) as gas phase reactions are unable to reproduce the large observed abundances (Roberts et al. 2004; Maret et al. 2005).

In pre-stellar cores, $\mathrm{HDCO} / \mathrm{H}_{2} \mathrm{CO}$ and $\mathrm{D}_{2} \mathrm{CO} / \mathrm{H}_{2} \mathrm{CO}$ abundance ratios up to $10 \%$ have been observed (Bacmann et al. 2003; Bergman et al. 2011). The observed deuterium fractionations increase with the increasing CO depletion, suggesting that the latter is a key parameter. Analogously, several formaldehyde and methanol isotopologues have been detected in Class 0 low mass protostars (Ceccarelli et al. 1998, 2001; Parise et al. 2002, 2004, 2006). However, unlike in prestellar cores, no correlation between the $\mathrm{CO}$ depletion and deuterium fractionation is observed, so that the deuteration process is thought to occur in the previous pre-collapse phase. In general, methanol is more enriched in deuterium than formaldehyde. In most of the observed sources, the simply deuterated molecule HDCO shows a deuterium fractionation between 13 and $20 \%$ whereas $\mathrm{CH}_{2} \mathrm{DOH}$ shows a fractionation between 37 and $65 \%$ (Parise et al. 2006). $\mathrm{CHD}_{2} \mathrm{OH}$ also shows a higher fractionation than $\mathrm{D}_{2} \mathrm{CO}$, by a factor of $\sim 2$. Finally, the deuteration process occurs more efficiently on the methyl group than on the hydroxyl group of methanol, as the $\left[\mathrm{CH}_{2} \mathrm{DOH}\right] /\left[\mathrm{CH}_{3} \mathrm{OD}\right]$ ratio has been ob-

\footnotetext{
${ }^{1}$ UJF-Grenoble 1 / CNRS-INSU, Institut de Planétologie et d'Astrophysique de Grenoble (IPAG) UMR 5274, Grenoble, F38041, France
}

served to be between 10 and 20 in a sample of low- and intermediate- mass protostars by Parise et al. (2006) and Ratajczak et al. (2011).

Recent experimental works have confirmed the synthesis of formaldehyde and methanol by hydrogenation of iced CO (Watanabe \& Kouchi 2002; Hidaka et al. 2007; Fuchs et al. 2009) and also highlighted the complex chemical evolution of $\mathrm{H}_{2} \mathrm{O}-\mathrm{CO}$ ices when they are exposed to $\mathrm{D}$ and $\mathrm{H}$ atoms. Indeed, Nagaoka et al. (2005), Nagaoka et al. (2007) and Hidaka et al. (2009) have shown that formaldehyde and methanol can be efficiently deuterated into $\mathrm{HDCO}, \mathrm{D}_{2} \mathrm{CO}, \mathrm{CH}_{2} \mathrm{DOH}$, $\mathrm{CHD}_{2} \mathrm{OH}$ and $\mathrm{CD}_{3} \mathrm{OH}$, when they are irradiated by $\mathrm{D}$ atoms. Conversely, abstractions of $\mathrm{D}$ can only occur on formaldehyde, and not on methanol. Therefore, formaldehyde isotopologues only can be hydrogenated to form back $\mathrm{HDCO}$ and $\mathrm{H}_{2} \mathrm{CO}$ if they are exposed to $\mathrm{H}$ atoms. The relative reaction rates deduced from these works show that $\mathrm{H}$ and $\mathrm{D}$ abstractions and substitutions on formaldehyde and methanol are as efficient as addition reactions (see also the theoretical calculations by Goumans 2011; Goumans et al. 2011). These processes could, therefore, largely increase the deuterium fractionations of formaldehyde and methanol after the complete depletion of $\mathrm{CO}$ and their formation on the surfaces.

In the past, astrochemical models have struggled to reproduce the observed deuteration ratios of formaldehyde and methanol. While it is now clear that $\mathrm{CO}$ depletion plays a major role in increasing the atomic $\mathrm{D} / \mathrm{H}$ ratio of the gas landing on the grain surfaces (Roberts et al. 2004), a full model coupling the gas and grain chemistry that simultaneously reproduces the observed formaldehyde and methanol deuteration is still missing. A previous attempt was carried out by Caselli et al. (2002) and Stantcheva \& Herbst (2003), who studied the formaldehyde and methanol deuteration as function of the atomic $\mathrm{D} / \mathrm{H}$ ratio, taken as a free parameter. However, as also emphasised by Parise et al. (2006), the use of a constant 
$[\mathrm{D}] /[\mathrm{H}]$ ratio is i) unable to predict all the deuterium fractionations at the same time and ii) is not necessarily correct, as formaldehyde and methanol may be formed on the grains at different times. Cazaux et al. (2011) have studied the deuteration of water and formaldehyde (and not methanol) by coupling gas phase and grain mantle chemical networks.

In this article, we re-consider the problem of the formaldehyde and methanol deuteration, using our gasgrain coupled model, GRAINOBLE, that takes into account $\mathrm{D}$ and $\mathrm{H}$ atoms addition, but also abstraction and substitution reactions. Our goal is to simultaneously reproduce the formaldehyde and methanol deuteration observed towards Class 0 protostars by considering their formation on interstellar grains only. Note that, once sublimated from ices, the abundance of these species is only slightly affected by gas phase reactions, as the typical chemical timescale $\left(\sim 10^{5} \mathrm{yr}\right.$, Charnley et al. 1997) is larger than the typical age of these objects $\left(\sim 10^{4}\right.$ yr, André et al. 2000). We will show that understanding how and when the observed deuteration occurs will also provide us with hints on the process itself and on the past history of the protostars.

\section{MULTILAYER MODELING OF DEUTERATED ICES}

\subsection{Description of the GRAINOBLE model}

For this study we use the GRAINOBLE model (Taquet et al. 2011, hereafter TCK11). Briefly, it is a gas-grain chemical model, based on the rate equations approach introduced by Hasegawa et al. (1992) for timedependent grain surface chemistry modeling. It considers the following four processes:

i) Accretion of gas phase species onto the grain surfaces as function of time.

ii) Thermal diffusion of adsorbed species. The hopping rate follows a Boltzmann function which depends on the diffusion energy $E_{d}$.

iii) Surface reactions via the Langmuir-Hinshelwood mechanism. The probability of the reaction is given by the exponential portion of the quantum mechanical probability for tunneling through a square barrier and therefore depends on the activation energy $E_{a}$ of the reaction. iv) Thermal evaporation caused by the thermal balance and by the cosmic-ray induced heating of the grains (Hasegawa \& Herbst 1993a). We ignored the photolytic process due to the cosmic-ray induced UV photons because they have a negligible influence on the abundance of formaldehyde and methanol in dark clouds $(5 \%$ at maximum, Cuppen et al. 2009).

We follow the mantle formation on grains with a multilayer approach in which the outermost layer only is reactive, while the mantle bulk remains inert. Unlike Hasegawa \& Herbst (1993b) and Garrod \& Pauly (2011) models, the trapping of particles into the bulk is performed one layer at a time, once the considered layer is filled.

\subsection{Chemical network}

We consider the accretion of gaseous $\mathrm{H}, \mathrm{D}, \mathrm{H}_{2}, \mathrm{HD}$, $\mathrm{D}_{2}, \mathrm{O}$, and $\mathrm{CO}$ onto grains.

We assume that formaldehyde, methanol and their deuterated isotopologues are formed via hydrogenation and deuteration addition reactions on molecules initiated by the accretion of $\mathrm{CO}$. Based on experimental and theoretical works (Hidaka et al. 2007; Watanabe \& Kouchi 2008; Andersson et al. 2011), the energy barriers of the $\mathrm{D}$ and $\mathrm{H}$ addition reactions involving $\mathrm{CO}$ and formaldehyde are very similar, so we assume them to be identical $\left(=E_{a}\right)$. Note that, however, given the high uncertainty in the value of $E_{a}$ (see TCK11), this is taken as a free parameter. In contrast, reactions involving a radical are barrierless.

Following the experimental works of Hidaka et al. (2009) and Nagaoka et al. (2007), we also include the abstraction and substitution reactions of $\mathrm{H}$ and $\mathrm{D}$ on formaldehyde and methanol according the scheme proposed by Watanabe \& Kouchi (2008) and Hidaka et al. (2009), and shown in Fig. 1]. We adopt the probability of each reaction following the relative rates deduced experimentally when they are available, as marked in the Figure. For the reactions that have not been derived by the experiments, we adopt the probability measured for the same isotopologue by analogy. For example, the reaction $\mathrm{D}_{2} \mathrm{CO}+\mathrm{D} \rightarrow \mathrm{CD}_{3} \mathrm{O}$ has a reaction rate equal to the $\mathrm{H}+\mathrm{CO}$ rate multiplied by $0.1(\mathrm{D}+\mathrm{CO})$ and 0.66 $\left(\mathrm{D}_{2} \mathrm{CO}+\mathrm{H}\right)$.

Even if most of water is likely formed during the translucent phase, and therefore before $\mathrm{H}_{2} \mathrm{CO}$ and $\mathrm{CH}_{3} \mathrm{OH}$ (see Oberg et al. 2011), our model also takes into account its formation, as it is in competition with formaldehyde and methanol formation according to TCK11. With respect to TCK11, we added the deuteration reactions and the path

$$
\mathrm{H}_{2}+\mathrm{OH} \rightarrow \mathrm{H}_{2} \mathrm{O}+\mathrm{H}
$$

which seems to be the most efficient reaction in molecular clouds, as suggested by Cuppen \& Herbst (2007).

Since the deuteration of formaldehyde and methanol on the grains depends on the atomic gas phase $\mathrm{D} / \mathrm{H}$ ratio, we are here particularly interested in the chemical network leading to the formation of atomic deuterium in the gas phase. We use the fractionation reactions introduced by Roberts \& Millar (2000); Roberts et al. (2004) to study the formation of $\mathrm{H}_{3}^{+}$isotopologues and atomic deuterium. The gas phase chemical network is shown in Figure 1. As shown by Flower et al. (2006), the ortho/para ratio (opr) of $\mathrm{H}_{2}$ can influence the deuteration of $\mathrm{H}_{3}^{+}$and consequently the atomic $\mathrm{D} / \mathrm{H}$ ratio, but only when it is $\geq 10^{-3}$. The available measures in cold gas indicate low $\mathrm{H}_{2}$ opr values $\left(<10^{-3}-10^{-2}\right.$, Dislaire et al. 2012, and references therein). We, therefore, did not consider this effect in this work.

\subsection{The physical model}

Rather than simulating the evolution from the diffuse cloud state to the pre-stellar core, we focus on the last stage of the evolution, when the material is already molecular and the density reaches the value $n_{H}$. The initial abundance of gas phase species $\left(\mathrm{H}, \mathrm{D}, \mathrm{HD}, \mathrm{D}_{2}, \mathrm{H}_{3}^{+}\right.$ isotopologues, ...) are given by the steady-state abundance, obtained solving the gas phase chemical network presented in $\S 2.2$ and considering the recombination of $\mathrm{H}$ and $\mathrm{D}$ onto grains. The abundance relative to $\mathrm{H}$ nuclei of the deuterium and $\mathrm{CO}$ reservoirs are $1.5 \times 10^{-5}$ and $4.75 \times 10^{-5}$. 

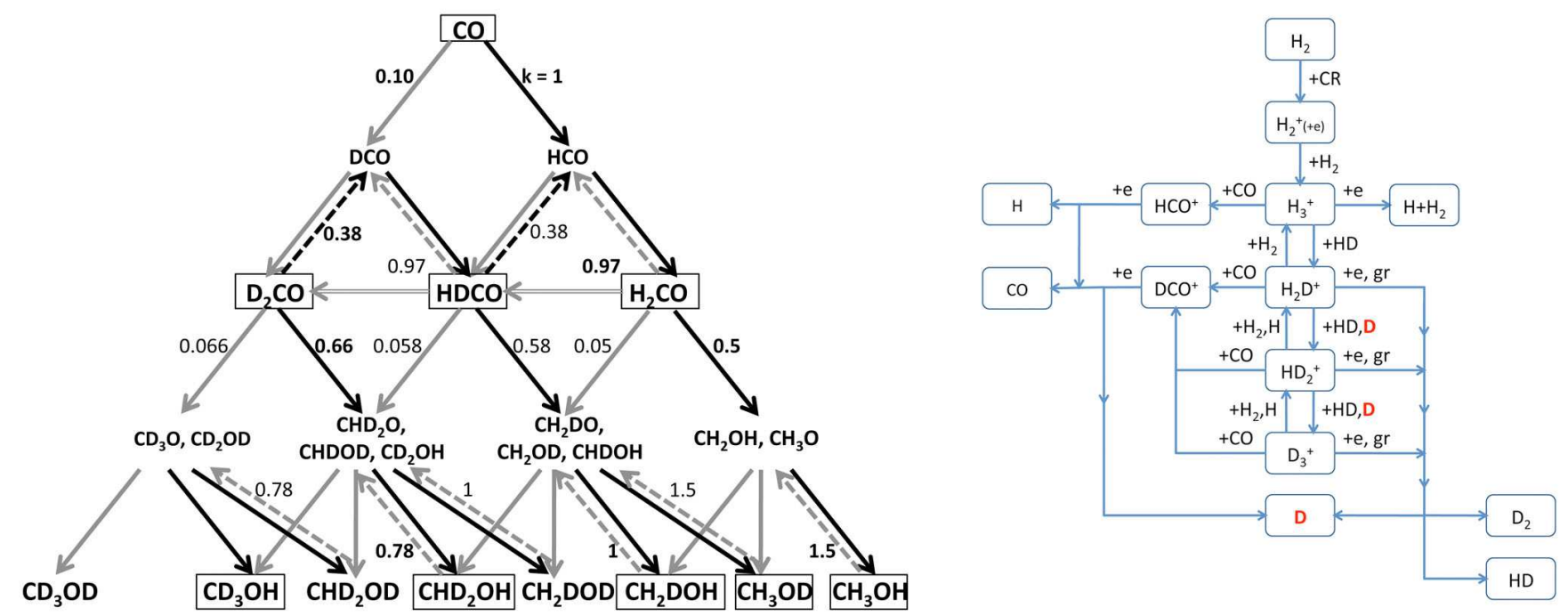

FIG. 1. - left) chemical network on grain mantles focusing on the formation of formaldehyde and methanol. Solid, dashed, and open arrows refer to addition, abstraction, and substitution reactions. Black arrows refer to reactions involving $\mathrm{H}$ while grey arrows represent reactions involving D. Finally, the bold values refer to the rates relative to the $\mathrm{H}+\mathrm{CO}$ reaction experimentally deduced by Nagaoka et al. (2007) and Hidaka et al. (2009) while non-bold values show the deduced relative rates by analogy with similar reactions. right) Chemical network in the gas phase leading to atomic deuterium formation.

We follow, then, the formation and evolution of the grain mantles during this phase, keeping the gas density $n_{H}$ constant. Briefly, as the time passes, CO freezes out onto the grain mantles, where it forms $\mathrm{H}_{2} \mathrm{CO}$ and $\mathrm{CH}_{3} \mathrm{OH}$ and their deuterated isotopologues. Therefore, the deuteration of the formaldehyde and methanol on the mantle primarily depends on the gaseous atomic $\mathrm{D} / \mathrm{H}$ ratio.

\subsection{Multi-parameter approach}

We use a multi-parameter approach as described in TCK11, to take into account the variation or the uncertainty of physical conditions and surface parameters.

Low mass pre-stellar cores are the place where most of formaldehyde and methanol are assumed to be formed. These objects show spatial distributions of densities of $\mathrm{H}$ nuclei between $10^{4}$ and $5 \times 10^{6} \mathrm{~cm}^{-3}$ and temperatures between 8 and $12 \mathrm{~K}$ (see Crapsi et al. 2007). Accordingly, we consider here four density values $\left(n_{H}=\right.$ $\left.10^{4}, 10^{5}, 10^{6}, 5 \times 10^{6} \mathrm{~cm}^{-3}\right)$ and three temperatures $\left(T_{g}=T_{d}=8,10,12 \mathrm{~K}\right)$. Besides, prestellar cores show an increase of the grain sizes caused by the coagulation process whose the efficiency increases with the density (Flower et al. 2005; Vastel et al. 2006). We adopt three grain sizes $a_{d}=0.1,0.2,0.3 \mu \mathrm{m}$, which affect the depletion rates and therefore the density of $\mathrm{H}$ and $\mathrm{D}$ in the gas phase.

As discussed in TCK11, the diffusion to desorption energy $E_{d} / E_{b}$ ratio mainly depends on the ice properties. We, therefore, vary $E_{d} / E_{b}$ between 0.5 and 0.8 , as suggested by experimental studies. The activation energy $E_{a}$ of the reactions involving $\mathrm{CO}$ and formaldehyde is also a free parameter. By comparing the model predictions with the observations of solid $\mathrm{CO}$ and methanol, we have deduced in TCK11 that $E_{a}$ must be lower than 1500 $\mathrm{K}$, so that we restrain the value of $E_{a}$ between 400 and $1400 \mathrm{~K}$. Light particles $\left(\mathrm{H}, \mathrm{D}, \mathrm{H}_{2}, \mathrm{HD}, \mathrm{D}_{2}\right)$ that accrete onto the ices have a binding energy distribution that depends on the ice properties, and the adsorption conditions of the adsorbate (see for example Hornekær et al.

2005). We therefore consider the binding energies relative to amorphous water ice of these light species as a free parameter whose values are: 400, 500, $600 \mathrm{~K}$.

As the abundance of the gaseous atomic oxygen in the pre-stellar cores is uncertain, we consider three values of oxygen abundance relative to $\mathrm{H}$ nuclei $X(\mathrm{O})_{i n i}: 10^{-8}$, $10^{-6}, 10^{-4}$.

Finally, in TCK11, we have shown that the site size $d_{s}$ and the porosity factor $F_{\text {por }}$ have a little impact on the chemical composition of the grain mantles, so we assume $d_{s}=3.1 \AA$ (Jenniskens et al. 1995), and $F_{\text {por }}=0$.

We run a grid of 2916 models in which we vary the parameters described above and listed in Table 1] For each density $n_{H}$, we compute the mean fractionation of each isotopologue with its 1 sigma standard deviation, following Wakelam et al. (2010).

TABLE 1

LIST OF PARAMETERS AND THE VALUES RANGE EXPLORED IN THIS WORK.

\begin{tabular}{cc}
\hline \hline Parameter & Values \\
\hline Density $n_{H}$ & $10^{4}-10^{5}-10^{6}-5 \times 10^{6} \mathrm{~cm}^{-3}$ \\
Temperature $T_{g}=T_{d}$ & $8-10-12 \mathrm{~K}$ \\
Grain size $a_{d}$ & $0.1-0.2-0.3 \mu \mathrm{m}$ \\
Energy ratio $E_{d} / E_{b}$ & $0.5-0.65-0.8$ \\
Binding energy $E_{b, \text { wat }}(\mathrm{H})$ & $400-500-600 \mathrm{~K}$ \\
Activation energy $E_{a}$ & $400-900-1400 \mathrm{~K}$ \\
Initial oxygen abundance $X(\mathrm{O})_{i n i}$ & $10^{-8}-10^{-6}-10^{-4}$ \\
\hline
\end{tabular}

\section{RESULTS}

\subsection{The $D / H$ gas phase ratio}

At first approximation, the steady state densities of $\mathrm{H}$ and $\mathrm{D}$, assumed as initial conditions, are roughly constant regardless the total density and their abundance relative to $\mathrm{H}$ nuclei, therefore, decrease with increasing $n_{H}$. At low densities, a significant fraction $(\sim 30 \%)$ of the deuterium reservoir is already in atomic form before the depletion of $\mathrm{CO}$ : the increase of the atomic $\mathrm{D} / \mathrm{H}$ ra- 
tio with the $\mathrm{CO}$ depletion will therefore be low. On the contrary, at high densities, only a negligible fraction of deuterium is in the atomic form at the beginning, so that the atomic $\mathrm{D} / \mathrm{H}$ ratio strongly increases with the $\mathrm{CO}$ depletion. The larger the density, the larger the gaseous atomic $\mathrm{D} / \mathrm{H}$ ratio increase with the $\mathrm{CO}$ depletion, as highlighted in Figure 2 which shows the evolution of the atomic $\mathrm{D} / \mathrm{H}$ as function of $\mathrm{CO}$ depletion, for $n_{H}$ equal to $10^{5}$ and $5 \times 10^{6} \mathrm{~cm}^{-3}$ respectively.

\subsection{Model with addition reactions only}

In this section, only addition reactions are considered on grain mantles (see §2.2). In this case, the deuteration of formaldehyde and methanol primarily depends on two factors, as illustrated in Fig. 22 i) The increase of the gas phase atomic $D / H$ ratio with the $C O$ depletion: the ratio increases with increasing $\mathrm{CO}$ depletion, namely with time, and with increasing density (see above); ii) When formaldehyde and methanol are formed: as explained in detail in TCK11, the increase of $n_{H}$ delays the formation of formaldehyde and methanol. In general, the two effects results in a larger deuteration of formaldehyde and methanol for larger densities and larger evolutionary times.

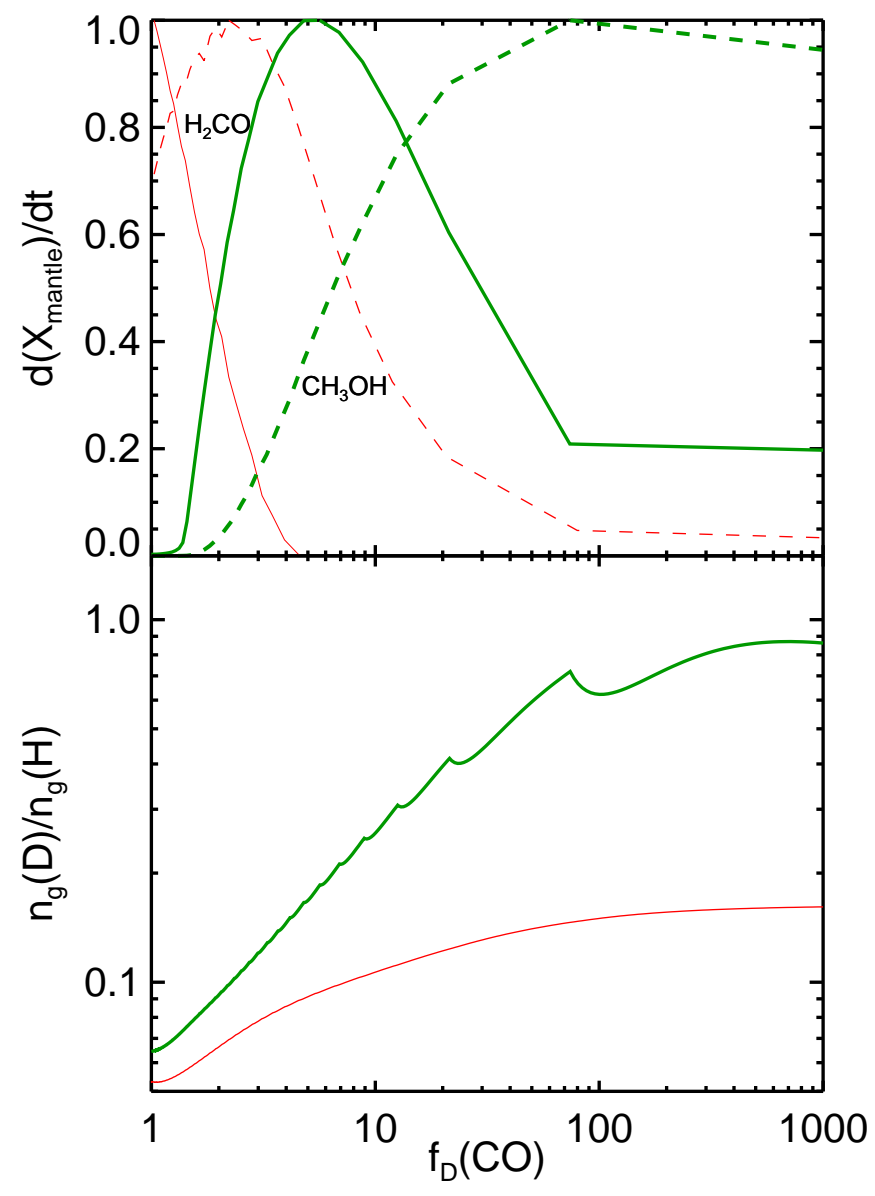

FIG. 2.- Normalized $\mathrm{H}_{2} \mathrm{CO}$ (solid lines) and $\mathrm{CH}_{3} \mathrm{OH}$ (dashed lines) formation rates (top panel) and gaseous atomic $\mathrm{D} / \mathrm{H}$ ratio (bottom panel) as function of the $\mathrm{CO}$ depletion factor $f_{D}(\mathrm{CO})=$ $n_{g}(\mathrm{CO}) / n_{g, \text { ini }}(\mathrm{CO})$, for $n_{H}=10^{5}$ (thin red lines), and $5 \times 10^{6}$ (thick green lines) $\mathrm{cm}^{-3}$. The spikes are caused by the multilayer nature of the mantle.
This is illustrated in Fig. 3, which shows the temporal evolution of the mean deuterium fractionations of iced formaldehyde and methanol along with their uncertainty, for different densities. The high density cases $\left(n_{H} \geq 10^{6} \mathrm{~cm}^{-3}\right)$ show large enhancements of the $\mathrm{CH}_{2} \mathrm{DOH} / \mathrm{CH}_{3} \mathrm{OH}$ and $\mathrm{HDCO} / \mathrm{H}_{2} \mathrm{CO}$ ratios with time, reaching the unity for the singly deuterated forms. No increase of the deuterium fractionation is, on the contrary, observed at low densities $\left(n_{H}=10^{4}-10^{5} \mathrm{~cm}^{-3}\right)$, the deuteration ratios never exceeding 0.02 . On the same figure, we report the range of observed values (from Parise et al. 2006). No model can reproduce the full set of observations. Indeed, the high density cases predict $\mathrm{CH}_{2} \mathrm{DOH} / \mathrm{CH}_{3} \mathrm{OH}$ and $\mathrm{HDCO} / \mathrm{H}_{2} \mathrm{CO}$ ratios in agreement with observations for times between $5 \times 10^{3}$ and $10^{6} \mathrm{yr}$. However, they cannot predict the observed high abundances of the doubly deuterated molecules. In addition, observations show that methanol is three times more deuterated than formaldehyde whereas the models of Fig. 3 predict a factor 1.5 at most. We conclude that this class of models fails to reproduce the observations.

\subsection{Abstraction and substitution reactions}

Figure 4 shows the temporal evolutions of formaldehyde and methanol deuterium fractionations when abstraction and substitution reactions are included. From Fig. 4 it is clear that the inclusion of the addition and substitution reactions strongly increase the deuterium fractionations, especially at high densities. For densities $\sim 5 \times 10^{6} \mathrm{~cm}^{-3}$, the model predicts fractionations of doubly-deuterated molecules compatible with observations, at time $\sim 5 \times 10^{3}$ yr. Unlike $\mathrm{CH}_{2} \mathrm{DOH}$, which shows an increase of deuteration compared to Fig. 3 and more particularly at longer timescales, the HDCO deuteration is not enhanced. This is due to the efficiency of deuterium abstraction on HDCO which allows the formation of $\mathrm{H}_{2} \mathrm{CO}$ whereas deuterium abstraction reactions on $\mathrm{CH}_{2} \mathrm{DOH}$, leading to $\mathrm{CH}_{3} \mathrm{OH}$ formation, are negligible. The observed $\left[\mathrm{CH}_{2} \mathrm{DOH}\right] /[\mathrm{HDCO}]$ ratio of 3 can now be predicted. Finally, because the abstraction reactions can only occur significantly on the methyl group of methanol and not on its hydroxyl group, the $\left[\mathrm{CH}_{2} \mathrm{DOH}\right] /\left[\mathrm{CH}_{3} \mathrm{OD}\right]$ ratio in enhanced. The observed ratio of 10-20 can also be predicted but at a larger time, $5-10 \times 10^{4} \mathrm{yr}$. We conclude that this class of models succeeds to reproduce all the observations simultaneously, with the exception of the $\left[\mathrm{CH}_{2} \mathrm{DOH}\right] /\left[\mathrm{CH}_{3} \mathrm{OD}\right]$ ratio, for a density of about $5 \times 10^{6} \mathrm{~cm}^{-3}$ and at a time of 5000 yr.

\section{DISCUSSION AND CONCLUSIONS}

Although multiply deuterated molecules in low mass protostars have been discovered and observed for a decade, published models have had difficulties in reproducing the observed abundances, especially those of formaldehyde and methanol (Parise et al. 2006). Both species are believed to be mainly synthesised and to be observed on the grain surfaces during the cold and dense pre-collapse phase (see Oberg et al. 2011) and that they are observed in the gas when they sublimate off the grain mantles upon heating from the central star. On the grains, $\mathrm{H}_{2} \mathrm{CO}$ and $\mathrm{CH}_{3} \mathrm{OH}$ are thought to be the result of the hydrogenation of iced CO. Their deuteration, 


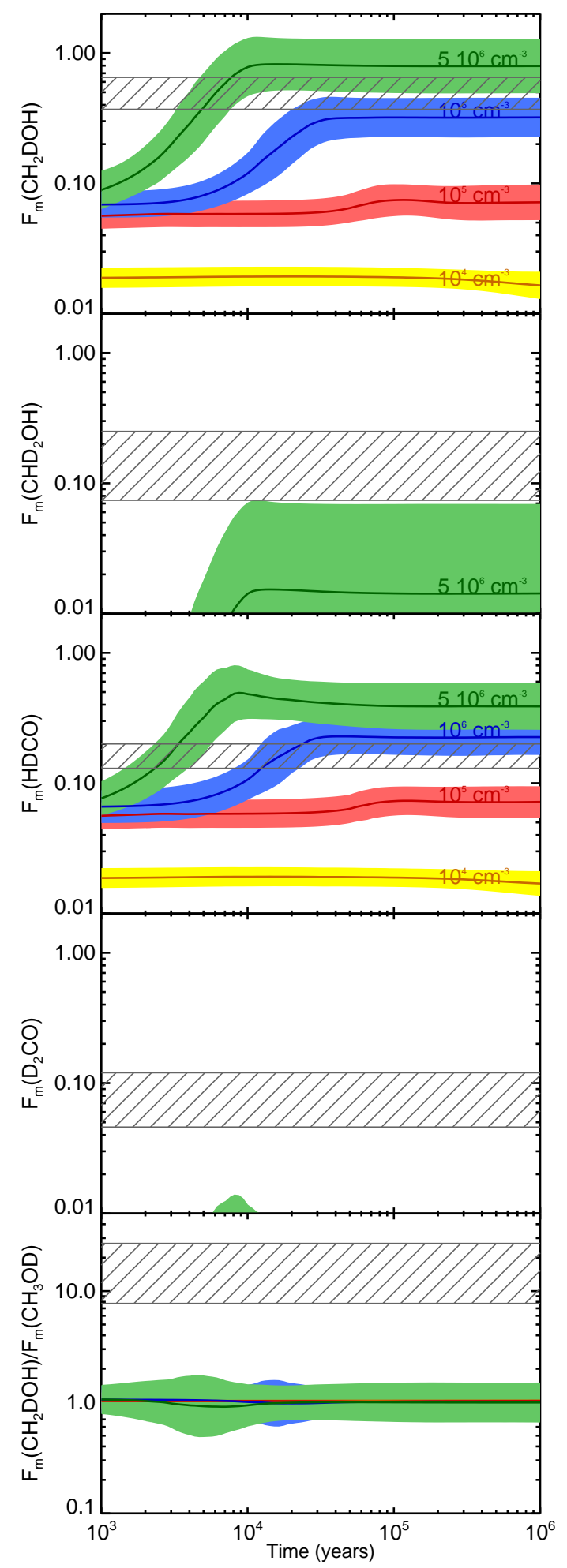

FIG. 3.- Mean deuterium fractionation of methanol and formaldehyde (solid lines) with their 1 sigma standard deviation (color levels) with time, obtained considering addition reactions only (§3.2). The hatched zones give the range of observed values (Parise et al. 2006).

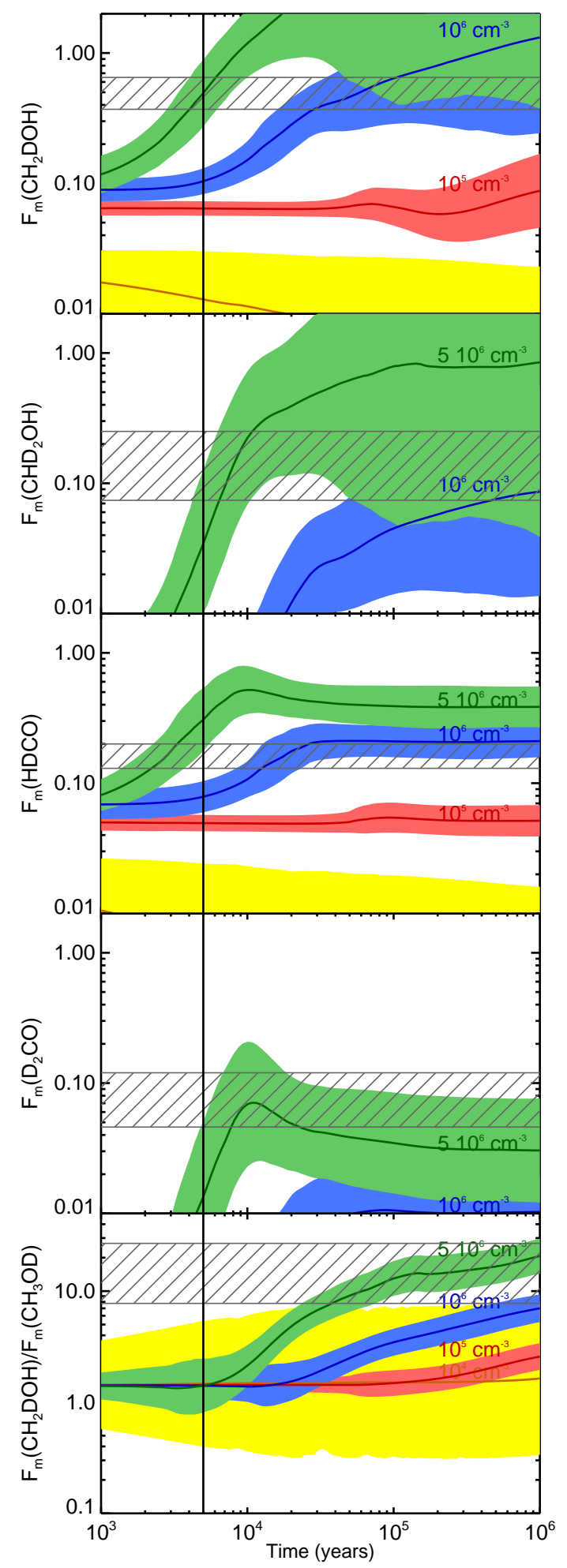

FIG. 4.- Mean deuterium fractionation of methanol and formaldehyde (solid lines) with their 1 sigma standard deviation (color levels) with time, obtained adding the abstraction and substitution reactions ( $\S 3.3)$. The hatched zones give the range of observed values (Parise et al. 2006). 
therefore, depends on when exactly the two species are formed and how. We have shown that, based on our grain surface model GRAINOBLE, formation of $\mathrm{H}_{2} \mathrm{CO}$ and $\mathrm{CH}_{3} \mathrm{OH}$ from addition reactions alone fails to predict the observed deuterium fractionation. In contrast, if D and $\mathrm{H}$ abstraction and substitution reactions are added, the GRAINOBLE model can reproduce simultaneously the observed values. Therefore, these processes are crucial and more laboratory experiments and theoretical computations are needed to better constrain their rates on the ices. Reproducing the observed $\left[\mathrm{CH}_{2} \mathrm{DOH}\right] /\left[\mathrm{CH}_{3} \mathrm{OD}\right]$ ratio remains a challenge, as it is still underestimated by the model. Previous studies have suggested that this may be caused by $\mathrm{D}$ and $\mathrm{H}$ exchanges on the ices during the sublimation phase (Rataiczak et al. 2009) or activated by photolysis processes (Weber et al. 2009) or on the gas phase (Osamura et al. 2004), all processes that would be inefficient in altering the other isotopologues (see Ratajczak et al. 2011, for a detailed discussion).

With the above exception, our model predicts the observed abundance ratios for high densities $\left(\sim 5 \times 10^{6}\right.$ $\left.\mathrm{cm}^{-3}\right)$ and for relatively short times $(\sim 5000 \mathrm{yr})$. We emphasise that this time corresponds only to the final stage at high density and not to the age of the condensation, which can be considerably larger. In fact, it is possible and even likely that the pre-stellar cores spend a long time in a less dense phase (e.g. Bergin \& Tafalla 2007). However, the comparison of the observed $\mathrm{H}_{2} \mathrm{CO}$ and $\mathrm{CH}_{3} \mathrm{OH}$ deuteration with our model predictions suggests that the last phase at high density is short, just a few thousands years. In other words, soon after the central density reaches $\sim 5 \times 10^{6} \mathrm{~cm}^{-3}$ the protostellar collapse starts. This is a nice illustration of how chemistry can help us to understand the past history of the protostar.

In conclusion, our work leads to two important results: 1) The pre-collapse phase may last hundred thousands of years: however, when the central density reaches $\sim 5 \times 10^{6} \mathrm{~cm}^{-3}$, the collapse starts in about a few thousands years. 2) $\mathrm{D}$ and $\mathrm{H}$ atoms abstraction and substitution reactions are crucial in the grain surface chemistry and should be incorporated into models. Thus, more experimental and theoretical works are therefore needed to better contrain their efficiency and therefore the timescale needed to reproduce the observations.

This work has been supported by l'Agence Nationale pour la Recherche (ANR), France (project FORCOMS, contracts ANR-08-BLAN-022).

\section{REFERENCES}

Andersson, S. and Goumans, T. P. M. and Arnaldsson, A. 2011 Chemical Physics Letters, 513, 31-36

André, P. and Ward-Thompson, D. and Barsony, M. 2000, Protostars and Planets IV, 59

Bacmann, A. and Lefloch, B. and Ceccarelli, C. and Steinacker, J. et al. 2003, ApJ, 585, L55-L58

Bergin, E. A. and Tafalla, M. 2007, ARA\&A, 45, 339-396

Bergman, p. and Parise, B. and Liseau, R. and Larsson, B. 2011, A\&A, 527, 39

Caselli, P. and Stantcheva, T. and Shalabiea, O. and Shematovich, V. I. and Herbst, E. 2002, Planetary and Space Science, 50, 1257-1266

Cazaux, S. and Caselli, P. and Spaans, M. 2011, ApJ, 741, L34

Ceccarelli, C. and Castets, A. and Loinard, L. and Caux, E. and Tielens, A. G. G. M. 1998, A\&A, 338, L43-46

Ceccarelli, C. and Loinard, L. and Castets, A. and Tielens, A. G. G. M. et al. 2001, A\&A, 372, 998-1004

Ceccarelli, C. and Caselli, P. and Herbst, E. and Tielens, A. G. G. M. and Caux, E. 2007, Protostars and Planets V Charnley, S. B. and Tielens, A. G. G. M. and Rodgers, S. D. ApJ, 1997, 482, L203

Crapsi, A. and Caselli, P. and Walmsley, M. C. and Tafalla, M. 2007, A\&A, 470, 221-230

Cuppen, H. M. and Herbst, E. 2007, ApJ, 668, 294

Cuppen, H. M. and van Dishoeck, E. F. and Herbst, E. and Tielens, A. G. G. M. 2009, A\&A, 508, 275

Dislaire, V. and Hily-Blant, P. and Faure, A. and Maret, S. and Bacmann, A. and Pineau Des Forêts, G. 2012, 537, A20

Flower, D. R. and Pineau Des Forêts, G. and Walmsley, C. M. 2005, A\&A, 436, 933-943

Flower, D. R. and Pineau Des Forêts, G. and Walmsley, C. M. 2006, A\&A, 449, 621629

Fuchs, G. W. and Cuppen, H. M. and Ioppolo, S. and Romanzin, C. and Bisschop, S. E. and Andersson, S. and van Dishoeck, E. F. and Linnartz, H. 2009, 505, 629-639

Garrod, R. T. and Pauly, T. 2011, ApJ, 735, 15

Goumans, T. P. M. 2011, MNRAS, 413, 2615-2620

Goumans, T. P. M. and Kaestner, J. 2011, J Phys Chem A, 115, 10767-10774

Hasegawa, T. I., Herbst, E. and Leung C. M. 1992, ApJ, 82, 167

Hasegawa, T. I. and Herbst, E. 1993, MNRAS, 261, 83-102

Hasegawa, T. I. and Herbst, E. 1993, MNRAS, 263, 589-606
Hidaka, H. and Kouchi, A. and Watanabe, N. 2007, Journal of Chemical Physics, 126, 204707

Hidaka, H. and Watanabe, M. and Kouchi, A. and Watanabe, N. 2009, ApJ, 702, 291-300

Hornekær, L. and Baurichter, A. and Petrunin, V. V. and Luntz, A. C. and Kay, B. D. and Al-Halabi, A. 2005, J. Chem. Phys., 122,12

Jenniskens, P., Blake, D. F., Wilson, M. A. et al. 1995, ApJ, 455, 389

Linsky, J. L. 2003, Space Science Reviews, 106, 49-60

Maret, S. and Ceccarelli, C. and Tielens, A. G. G. M. and Caux, E. and Lefloch, B. and Faure, A. and Castets, A. and Flower, D. R. 2005, A\&A, 442, 527-538

Nagaoka, A. and Watanabe, N. and Kouchi, A. 2005, ApJ, 624, L29-L32

Nagaoka, A. and Watanabe, N. and Kouchi, A. 2007, J. Phys. Chem. A, 111, 3016-3028

Öberg, K. I. and Boogert, A. C. A. and Pontoppidan, K. M. and van den Broek, S. and van Dishoeck, E. F. and Bottinelli, S. and Blake, G. A. and Evans, II, N. J. 2011, ApJ, 740, 109

Osamura, Y. and Roberts, H. and Herbst, E. 2004, A\&A, 421, 1101

Parise, B. and Ceccarelli, C. and Tielens, A. G. G. M. and Herbst, E. and Lefloch, B. and Caux, E. and Castets, A. and Mukhopadhyay, I. and Pagani, L. and Loinard, L. 2002, A\&A, 393, L49-L53

Parise, B. and Castets, A. and Herbst, E. and Caux, E. and Ceccarelli, C. and Mukhopadhyay, I. and Tielens, A. G. G. M. 2004, A\&A, 416, 159-163

Parise, B. and Ceccarelli, C. and Tielens, A. G. G. M. and Castets, A. and Caux, E. and Lefloch, B. and Maret, S. 2006, A\&A, 453, 949-958

Ratajczak, A. and Quirico, E. and Faure, A. and Schmitt, B. and Ceccarelli, C. 2009, A\&A, 496, L21-L24

Ratajczak, A. and Taquet, V. and Kahane, C. and Ceccarelli, C. and Faure, A. and Quirico, E. 2011, A\&A, 528, L13

Roberts, H. and Millar, T. J. 2000, A\&A, 361, 388-398

Roberts, H. and Herbst, E. and Millar, T. J. 2004, A\&A, $424,905-917$

Stantcheva, T. and Herbst, E. 2003, MNRAS, 340, 983-988

Taquet, V., Ceccarelli, C., and Kahane, C. 2011, A\&A, accepted Tielens, A. G. G. M. and Hagen, W. 1982, A\&A, 114, 245-260 
Vastel, C. and Caselli, P. and Ceccarelli, C. and Phillips, T. et al. 2006, ApJ, 645, 1198-1211

Vidali, G. and Ihm, G. and Kim, H. and Cole, M. 1991, Surface Science Reports, 12, 4, 135-181

Wakelam, V. and Herbst, E. and Le Bourlot, J. and Hersant, F. and Selsis, F. and Guilloteau, S. 2010, A\&A, 517, 21
Watanabe, N. and Kouchi, A. 2002, ApJ, 571, L73

Watanabe, N. and Kouchi, A. 2008, Progress in Surface Science, 83, 439-489

Weber, A. S. and Hodyss, R. and Johnson, P. V. and Willacy, K. and Kanik, I. 2009, ApJ, 703, 10300 